\title{
(6) OPEN ACCESS \\ Are brief interventions to increase physical activity cost-effective? A systematic review
}

\author{
Vijay GC, ${ }^{1}$ Edward CF Wilson, ${ }^{1,2}$ Marc Suhrcke, ${ }^{1,3,4}$ Wendy Hardeman, ${ }^{5}$ \\ Stephen Sutton, ${ }^{5}$ on behalf of the VBI Programme Team
}

\begin{abstract}
- Additional material is published online only. To view please visit the journal online (http://dx.doi.org/10.1136/ bjsports-2015-094655).

${ }^{1}$ Health Economics Group, Norwich Medical School, University of East Anglia, Norwich Research Park, Norwich, UK

${ }^{2}$ Cambridge Centre for Health Services Research, Institute of Public Health, University of Cambridge School of Clinica Medicine, Cambridge, UK ${ }^{3}$ UKCRC Centre for Diet and Activity Research (CEDAR), University of Cambridge School of Clinical Medicine, Cambridge, UK

${ }^{4}$ Centre for Health Economics, University of York, York, UK ${ }^{5}$ Behavioural Science Group, Primary Care Unit, Institute of Public Health, University of Cambridge School of Clinical Medicine, Cambridge, UK
\end{abstract}

\section{Correspondence to} Vijay GC, Health Economics Group, Norwich Medical School, University of East Anglia, Norwich Research Park, Norwich NR4 7TJ, UK; vijay.gc@uea.ac.uk

Accepted 27 July 2015 Published Online First 5 October 2015
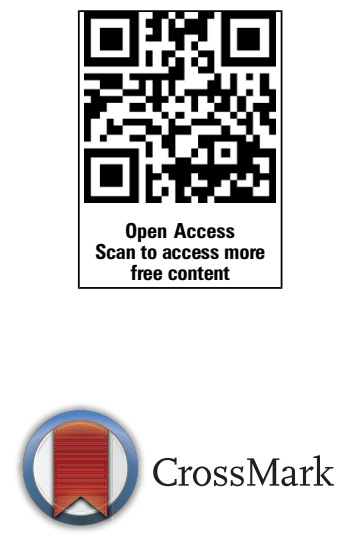

To cite: GC V, Wilson ECF, Suhrcke $\mathrm{M}$, et al. Br I Sports Med 2016;50:408-417.

\section{ABSTRACT}

Objective To determine whether brief interventions promoting physical activity are cost-effective in primary care or community settings.

Design Systematic review of economic evaluations. Methods and data sources We searched MEDLINE, EMBASE, PsycINFO, CINAHL, EconLit, SPORTDiscus, PEDro, the Cochrane library, National Health Service Economic Evaluation Database and the Cost-Effectiveness Analysis Registry up to 20 August 2014. Web of Knowledge was used for cross-reference search. We included studies investigating the cost-effectiveness of brief interventions, as defined by National Institute for Health and Care Excellence, promoting physical activity in primary care or the community. Methodological quality was assessed using Drummond's checklist for economic evaluations. Data were extracted from individual studies fulfilling selection criteria using a standardised pro forma. Comparisons of cost-effectiveness and cost-utility ratios were made between studies.

Results Of 1840 identified publications, 13 studies fulfilled the inclusion criteria describing 14 brief interventions. Studies varied widely in the methods used, such as the perspective of economic analysis, intervention effects and outcome measures. The incremental cost of moving an inactive person to an active state, estimated for eight studies, ranged from $\mathrm{f} 96$ to $\mathrm{f986}$. The cost-utility was estimated in nine studies compared with usual care and varied from $\mathrm{f} 57$ to $£ 14002$ per quality-adjusted life year; dominant to £6500 per disability-adjusted life year; and £15 873 per life years gained.

Conclusions Brief interventions promoting physical activity in primary care and the community are likely to be inexpensive compared with usual care. Given the commonly accepted thresholds, they appear to be costeffective on the whole, although there is notable variation between studies.

Physical inactivity leads to an increased risk of developing over 20 health conditions, including coronary heart disease (CHD), cancer, type 2 diabetes and stroke. ${ }^{12}$ In developed countries, these diseases and conditions attributable to physical inactivity account for $1.5-3 \%$ of total direct healthcare costs. ${ }^{3}$ In 2006-2007, the direct costs to the UK National Health Service (NHS) from treating the consequences of physical inactivity were estimated to be $£ 0.9$ billion. ${ }^{4}$ Physical activity not only improves physical health but also contributes to mental wellbeing. ${ }^{5}$ Incorporation of physical activity into individual lifestyles is known to lead to health benefits such as reduced risk of CHD, stroke and type 2 diabetes and the risk of premature death. ${ }^{67}$
In recent years, there has been substantial emphasis on efforts to promote physical activity along the continuum of individual-level and population-based interventions. ${ }^{8}$ The UK's Chief Medical Officers recommend that adults undertake at least $150 \mathrm{~min}$ of moderate-intensity activity each week. ${ }^{9}$ Despite the well-reported health and economic benefits of physical activity, results from the 2008 Health Survey for England, using selfreported measures, showed that only $39 \%$ of men and $29 \%$ of women achieved the recommended levels, ${ }^{10}$ a situation that had not improved by the time of the 2012 survey. $^{11}$

This led to a substantial policy interest to raise the overall physical activity in the general population. ${ }^{12}$ A wide range of interventions increase physical activity across the life course. ${ }^{13}{ }^{14}$ The National Institute for Health and Care Excellence (NICE) has published multiple pieces of guidance on physical activity promotion. In 2006, NICE published a guideline on four commonly used methods to increase physical activity (brief interventions in primary care, exercise referral schemes, pedometers and community-based exercise programmes for walking and cycling) ${ }^{15}$ supported by an economic analysis. ${ }^{16}$ More recently, NICE produced specific guidance on approaches to increase walking and cycling, ${ }^{17}$ and updated guidance on brief advice for adults in primary care ${ }^{18}$ and exercise referral schemes. ${ }^{19}$

There is an enthusiasm within government at national and local levels to support a wide variety of initiatives to promote physical activity, and reap the health, social and economic benefits. ${ }^{12}$ In times of particular budgetary restraint, there is an obvious need to look for low-cost initiatives. Brief interventions may be such a solution as they require less resources compared with more intensive interventions. Although they may have a small effect on an individual's behaviour, if sustained they could potentially have a significant population health impact. ${ }^{16}$

Current intervention strategies based in primary care or the community have provided the evidence that they can effectively increase physical activity. $^{20-22}$ Moreover, recent systematic reviews and meta-analyses of randomised and non-randomised trials have shown that physical activity interventions such as brief advice, exercise on prescription and physician counselling significantly increase physical activity behaviour and fitness in the longer term. ${ }^{23} 24$

Although brief interventions are effective in promoting physical activity, for policymakers and health funders it is necessary to know whether they 
are cost-effective to set priorities and allocate finite public funds. Previous reviews of economic evaluations of physical activity interventions suggest that most of the current intervention strategies are cost-effective. ${ }^{25-28}$ However, these reviews evaluated the cost-effectiveness of physical activity in general, included intensive interventions such as supervised exercise sessions, workplace interventions and only covered the literature to 2010. In addition, the lack of economic evidence for brief interventions in physical activity has been recognised. ${ }^{29}{ }^{30}$ Thus, we tailored our search to a specific question, that is, "What do we currently know about the cost-effectiveness of brief interventions delivered in primary care or community settings to increase physical activity?'

\section{METHODS}

\section{Definitions}

For the purpose of this review, we used the NICE definition of brief interventions: ${ }^{15}$ "Brief interventions involve opportunistic advice, discussion, negotiation or encouragement. They are commonly used in many areas of health promotion, and are delivered by a range of primary and community care professionals. The interventions vary from basic advice to more extended, individually-focused attempts to identify and change factors that influence activity levels". Brief interventions are typically conducted in face-to-face sessions with or without the addition of written materials, ranging from a single session of short duration $(5-30 \mathrm{~min})$ to multiple brief sessions or follow-ups. ${ }^{31}$

\section{Data sources}

We searched for articles published up to 20 August 2014 using the electronic databases MEDLINE, EMBASE, PsycINFO, CINAHL, EconLit, SPORTDiscus, Physiotherapy Evidence Database (PEDro), the Cochrane library, the NHS Economic Evaluation Database and the Tufts Cost-Effectiveness Analysis (CEA) Registry. In addition, the references of retrieved articles were examined manually after reviewing the title and abstracts to identify any economic studies alongside clinical trials and other pertinent studies. A cross-reference search was done using Web of Knowledge, and free text searching was performed using Google scholar. The search consisted of keywords and $\mathrm{MeSH}$ terms related to physical activity, exercise or fitness, brief or minimal intervention, and economic or cost analysis and was limited to the English language. Details of the search strategy used are described in online supplementary appendix 1.

\section{Study selection}

Studies were eligible for inclusion if they met the following three criteria:

1. Type of study-economic analyses alongside randomised controlled trials (RCTs) or non-experimental designs, or modelling studies of physical activity interventions which were based in either primary care or the community. Comparators could include usual care or other interventions.

2. Type of intervention-(a) interventions involving verbal advice, encouragement, negotiation or discussion, delivered face-to-face in a single session or multiple brief sessions, with or without additional non-face-to-face contacts (eg, leaflets or phone calls) or (b) interventions that were reported as 'brief' or 'minimal', and aimed to increase physical activity or fitness levels (or both) at the individual level (ie, brief interventions delivered to individuals or groups). Interventions were included if they were either the primary focus of the study or one of the comparator interventions, because physical activity interventions are often used in conjunction with or compared to other types of interventions (such as combined physical activity and dietary advice) for their physical well-being benefits.

3. Study populations-inactive adults aged 16 years or over. We excluded interventions where study populations were targeted or selected on the basis of pre-existing disease conditions (eg, osteoarthritis) because these populations require specialised interventions.

\section{Data extraction and analysis}

Initial screening of titles and abstracts against inclusion criteria was undertaken by one researcher (VGC) and potentially relevant articles were retrieved. Abstracted data were double checked by a second researcher (EW) and, if necessary, amendments were made after discussion. If there was insufficient information in the article on cost-effectiveness, we contacted the corresponding author. Included studies were assessed for methodological quality using Drummond's checklist ${ }^{32}$ for assessing economic evaluations. The checklist considers the following aspects: study question, selection of alternatives, form of economic evaluation, data collection (effectiveness data), benefit measurement and valuation, costing, modelling, allowance for uncertainty and presentation of results (table 1). On the basis of the number of Drummond's checklist criteria met, a rating of 'high' (9-10), 'good' (7-8), 'fair' (5-6) or 'poor' (1-4) was assigned.

We used a standardised pro forma to extract data from full texts on the type of economic analysis and perspective, interventions and comparison, participants, follow-up duration, outcome and cost-effectiveness results. To compare the economic results of the individual studies, we converted all costs to 2011 pounds sterling $(£)$ by applying the gross domestic product deflator index (GDP values) and purchasing power parities conversion rates using the Campbell \& Cochrane Economics Methods Group (CCEMG)-Evidence for Policy and Practice Information and Coordinating Centre (EPPI-Centre) Cost Converter (V1.4). ${ }^{33}{ }^{34}$ Cost-effectiveness results were grouped into those reporting intermediate outcomes such as the incremental cost of moving one inactive adult to an active category (ie, meeting the physical activity recommendations) ${ }^{9}$ and those reporting final outcomes such as the incremental cost per incremental quality-adjusted life year (QALY) gained, disability-adjusted life year (DALY) averted or life years gained (LYG).

\section{RESULTS}

\section{Literature search}

The search identified 1840 potentially relevant articles. An additional four articles were identified through reference lists and grey literature search. Following the removal of duplicates and the review of titles and abstracts, we assessed the full text of 28 articles. Thirteen met the inclusion criteria and were included in the review (figure 1). Five studies originated from the UK (one of them in Scotland), ${ }^{35-39}$ four from New Zealand, ${ }^{40-43}$ two from Australia, ${ }^{44}{ }^{45}$ and one each from Sweden ${ }^{46}$ and the Netherlands. ${ }^{47}$

Of the 15 excluded studies, six reported more intensive interventions and/or did not include a face-to-face component (eg, supervised exercise sessions, mail or telephone contact) $;^{48-53}$ four included a combined intervention targeting multiple behaviours (eg, physical activity and diet) $;^{54-57}$ two were exercise referral schemes (referral to a physical activity specialist or service); $;^{58} 59$ two did not report a physical activity or cost-effectiveness outcome ${ }^{60} \quad 61$ and one study evaluated the 
Table 1 Critical appraisal of included economic papers using Drummond et al's $\mathrm{s}^{32}$ checklist

\begin{tabular}{|c|c|c|c|c|c|c|c|c|c|c|}
\hline Study & $\begin{array}{l}\text { Well-defined } \\
\text { question } \\
\text { posed in } \\
\text { answerable } \\
\text { form }\end{array}$ & $\begin{array}{l}\text { Comprehensive } \\
\text { description of the } \\
\text { competing } \\
\text { alternatives given }\end{array}$ & $\begin{array}{l}\text { Effectiveness } \\
\text { of the } \\
\text { programme or } \\
\text { service } \\
\text { established }\end{array}$ & $\begin{array}{l}\text { All important and } \\
\text { relevant costs and } \\
\text { consequences for } \\
\text { each alternative } \\
\text { identified }\end{array}$ & $\begin{array}{l}\text { Costs and } \\
\text { consequences } \\
\text { measured accurately } \\
\text { in appropriate } \\
\text { physical units }\end{array}$ & $\begin{array}{l}\text { Cost and } \\
\text { consequences } \\
\text { valued credibly }\end{array}$ & $\begin{array}{l}\text { Costs and } \\
\text { consequences } \\
\text { adjusted for } \\
\text { differential timing }\end{array}$ & $\begin{array}{l}\text { Incremental } \\
\text { analysis of costs } \\
\text { and consequences } \\
\text { of alternatives } \\
\text { performed }\end{array}$ & $\begin{array}{l}\text { Allowance made } \\
\text { for uncertainty in } \\
\text { the estimates of } \\
\text { cost and } \\
\text { consequences }\end{array}$ & $\begin{array}{l}\text { Presentation and } \\
\text { discussion of study } \\
\text { results included all } \\
\text { issues of concerns } \\
\text { to users }\end{array}$ \\
\hline Anokye et $\left.a\right|^{35}$ & Yes & Yes & Yes & Yes & Yes & Yes & Yes & Yes & $\begin{array}{l}\text { Yes, probabilistic } \\
\text { sensitivity analysis }\end{array}$ & Yes \\
\hline Boehler et a $\beta^{36}$ & Yes & Yes & Yes & Yes & Yes & Yes & Not relevant & Yes & $\begin{array}{l}\text { Yes, probabilistic } \\
\text { sensitivity analysis }\end{array}$ & Yes \\
\hline Cobiac et $a l^{44}$ & Yes & Yes & Yes & Yes & Yes & Yes & Yes & Yes & $\begin{array}{l}\text { Yes, probabilistic } \\
\text { sensitivity analysis }\end{array}$ & Yes \\
\hline Dalziel et $a l^{40}$ & Yes & Yes & Yes & $\begin{array}{l}\text { Only included } \\
\text { programme costs- } \\
\text { cost of downstream } \\
\text { events not included } \\
\text { eg, CVD, diabetes }\end{array}$ & Yes & $\begin{array}{l}\text { Not all sources } \\
\text { cited (eg, source } \\
\text { for overhead } \\
\text { costs) }\end{array}$ & Yes & Yes & $\begin{array}{l}\text { Yes, probabilistic } \\
\text { sensitivity analysis }\end{array}$ & Yes \\
\hline Elley et $a{ }^{42}$ & Yes & Yes & Yes & Yes & Yes & $\begin{array}{l}\text { Not all sources } \\
\text { cited (eg, source } \\
\text { for overhead } \\
\text { costs) }\end{array}$ & Yes & $\begin{array}{l}\text { Yes but excluded lost } \\
\text { productivity from } \\
\text { calculation (reason } \\
\text { given by authors) }\end{array}$ & $\begin{array}{l}\text { Yes, one way } \\
\text { sensitivity analysis }\end{array}$ & Yes \\
\hline Elley et $a{ }^{41}$ & Yes & Yes & Yes & Yes & Yes & Yes & $\begin{array}{l}\text { Mostly; second year } \\
\text { costs not } \\
\text { discounted }\end{array}$ & Yes & $\begin{array}{l}\text { Yes, one way } \\
\text { sensitivity analysis } \\
\text { and hypothesis test } \\
\text { but of geometric } \\
\text { means }\end{array}$ & Yes \\
\hline $\begin{array}{l}\text { Gulliford } \\
\text { et }\left.a\right|^{37}\end{array}$ & Yes & Insufficient detail & Yes & Yes & Yes & Insufficient detail & Yes & Yes & $\begin{array}{l}\text { Yes, probabilistic } \\
\text { sensitivity analysis }\end{array}$ & Yes \\
\hline Leung et $a l^{43}$ & Yes & Yes & Yes & Yes & Yes & Yes & Yes & Yes & $\begin{array}{l}\text { Yes, probabilistic } \\
\text { sensitivity analysis }\end{array}$ & Yes \\
\hline $\begin{array}{l}\text { Lindgren } \\
\text { et } a^{46}\end{array}$ & Yes & $\begin{array}{l}\text { Yes but hypothetical } \\
\text { intervention }\end{array}$ & Yes & Yes & Yes & $\begin{array}{l}\text { Authors state } \\
\text { time and travel } \\
\text { costs not } \\
\text { included }\end{array}$ & Yes & Yes & $\begin{array}{l}\text { Probabilistic } \\
\text { sensitivity analysis } \\
\text { and scenario } \\
\text { analysis }\end{array}$ & Yes \\
\hline Over et al ${ }^{47}$ & Yes & Yes & Yes & Insufficient detail & Insufficient detail & $\begin{array}{l}\text { Not all sources } \\
\text { cited (cost, } \\
\text { utility) }\end{array}$ & Yes & Yes & $\begin{array}{l}\text { Yes, probabilistic } \\
\text { sensitive analysis }\end{array}$ & Yes \\
\hline Pringle et $a l^{38}$ & Yes & $\begin{array}{l}\text { Unclear if usual } \\
\text { practice and all the } \\
\text { relevant comparators } \\
\text { included, although } \\
\text { full details provided } \\
\text { in referenced sources }\end{array}$ & $\begin{array}{l}\text { Insufficient } \\
\text { detail about } \\
\text { source of study }\end{array}$ & $\begin{array}{l}\text { Out-of-pocket } \\
\text { expenditure excluded }\end{array}$ & Yes & Yes & Yes & Yes & $\begin{array}{l}\text { Yes, one way } \\
\text { sensitivity analysis }\end{array}$ & $\begin{array}{l}\text { Minimal information } \\
\text { about economic } \\
\text { model and sensitivity } \\
\text { analysis, although } \\
\text { references to more } \\
\text { details are provided }\end{array}$ \\
\hline Shaw et $a^{39}$ & Yes & Yes & Yes & $\begin{array}{l}\text { Restricted to } \\
\text { intervention costs }\end{array}$ & Yes & $\begin{array}{l}\text { Sources cited but } \\
\text { not clear which } \\
\text { relates to which } \\
\text { unit cost }\end{array}$ & $\begin{array}{l}\text { Not applicable to } \\
\text { within-trial } \\
\text { analysis, threshold } \\
\text { analysis for } f / Q A L Y \\
\text { does not appear to } \\
\text { take discounting } \\
\text { into account }\end{array}$ & Yes & $\begin{array}{l}\text { Mentioned but not } \\
\text { described in detail }\end{array}$ & $\begin{array}{l}\text { Discussion limited by } \\
\text { broad scope of the } \\
\text { paper (qualitative } \\
\text { and economic } \\
\text { analysis in one } \\
\text { paper) }\end{array}$ \\
\hline Sims et $a l^{45}$ & Yes & Yes & Yes & Yes & Yes & $\begin{array}{l}\text { Not all sources } \\
\text { cited }\end{array}$ & $\begin{array}{l}\text { Not clearly } \\
\text { mentioned }\end{array}$ & Yes & $\begin{array}{l}\text { Yes, univariate } \\
\text { sensitivity analysis }\end{array}$ & Yes but not in detail \\
\hline
\end{tabular}


Figure 1 PRISMA flow diagram of study selection.

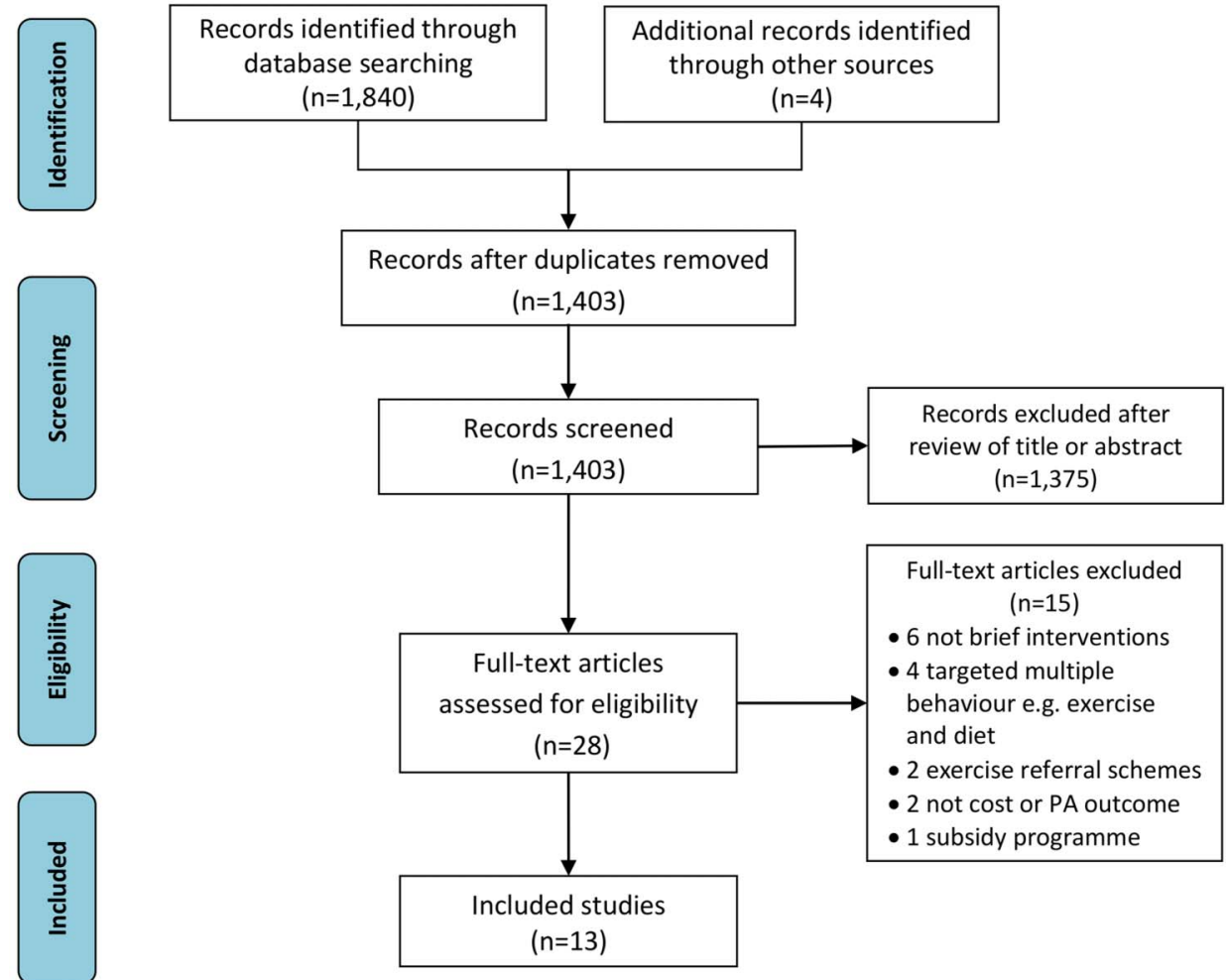

cost-effectiveness of a subsidy programme for general practitioner (GP) involvement in physical activity. ${ }^{62}$

\section{Characteristics of included studies}

Across the 13 studies, 30 intervention strategies or scenarios were evaluated, of which 14 met the definition of brief interventions. These were grouped into: brief exercise advice, exercise on prescription, pedometers and motivational interventions (table 2).

Four studies performed economic evaluation alongside controlled trials, ${ }^{39}{ }^{41-43}$ and two were based on quasi-experimental designs. ${ }^{3638}$ Seven studies performed economic modelling using data from a single trial, ${ }^{40446}$ a meta-analysis of RCTs, ${ }^{37} 4447$ a systematic review of randomised and observation studies, ${ }^{35}$ or a cross-sectional and observational study. ${ }^{45}$ Four studies reported intermediate outcomes in terms of the cost of making one additional inactive person active; ${ }^{36} 394142$ five reported final outcomes in terms of incremental cost per DALY ${ }^{44}$ or QALY $^{40} 47$ or $\mathrm{LYG}^{46}$ or incremental net health benefit $(\mathrm{NHB})^{37}$ and four reported both intermediate and cost-utility outcomes. ${ }^{35} 384345$

The 13 studies that met the inclusion criteria are described in table 3. The follow-up of RCTs ranged from 12 months to 2 years. One study had a short follow-up ( 3 months). ${ }^{36}$ Nine studies were of good or high quality. Modelling studies adopted a lifetime horizon. The measurement of physical activity was different across studies. The studies reporting a time-based outcome used a target of $\geq 5 \times 30 \mathrm{~min} /$ week of moderate or $\geq 60 \mathrm{~min} /$ week of vigorous-intensity activity. In pedometer-based interventions, a common threshold measure used was $\geq 10000$ steps/day except for one study ${ }^{39}$ which used a target of a weekly increase of $\geq 15000$ steps. Six studies ${ }^{36} 39-4245$ reported the duration of brief interventions (face-to-face), which lasted from 4 to $30 \mathrm{~min}$. Most (10) of the included studies evaluated each intervention in comparison with usual care or current practice, which approximates a 'do nothing' scenario.

\section{Studies reporting intermediate outcomes} was estimated for eight studies and ranged from $£ 96$ to $£ 986$ (figure 2). An 'active script programme' (an organised approach to exercise counselling by GPs) evaluated by Sims et $a l^{45}$ appeared to be the most cost-effective of the interventions considered ( $₫ 96$ per additional active participant compared with usual care). Boehler $e t a l^{36}$ evaluated the cost-effectiveness of two recruitment strategies for the delivery of brief exercise advice (disease register screening vs opportunistic patient
The cost of converting one inactive adult to an 'active' category

Table 2 Overview of interventions

\begin{tabular}{|c|c|c|c|c|}
\hline Interventions & Number & Short description & $\begin{array}{l}\text { Source of effectiveness } \\
\text { data }\end{array}$ & References \\
\hline Exercise advice & 5 & Brief exercise advice or counselling by a GP or trained health professionals & $\mathrm{CS}, \mathrm{MA}, \mathrm{OS}, \mathrm{RCT}$ & $35-37,45,46$ \\
\hline Exercise on prescription & 4 & Verbal and written physical activity advice by a GP or practice nurse & $\mathrm{MA}, \mathrm{RCT}$ & $40-42,44$ \\
\hline Pedometers & 4 & $\begin{array}{l}\text { Pedometer-based physical activity counselling with a step-related goal or walking } \\
\text { programme }\end{array}$ & $\mathrm{MA}, \mathrm{RCT}$ & $39,43,44,47$ \\
\hline $\begin{array}{l}\text { Motivational } \\
\text { interventions }\end{array}$ & 1 & Motivational interviews to increase physical activity & Pre-post intervention & 38 \\
\hline
\end{tabular}


Table 3 Characteristics of included studies

\begin{tabular}{|c|c|c|c|c|c|c|c|c|c|}
\hline $\begin{array}{l}\text { Study, } \\
\text { setting, } \\
\text { quality }\end{array}$ & $\begin{array}{l}\text { Objective; economic } \\
\text { perspective, cost year }\end{array}$ & $\begin{array}{l}\text { Study type; } \\
\text { economic } \\
\text { analysis type }\end{array}$ & Interventions compared & Participants & $\begin{array}{l}\text { Follow-up } \\
\text { duration }\end{array}$ & $\begin{array}{l}\text { Definition of } \\
\text { physically } \\
\text { active person }\end{array}$ & $\begin{array}{l}\text { Mean time to } \\
\text { deliver } \\
\text { intervention per } \\
\text { person }\end{array}$ & $\begin{array}{l}\text { Cost of converting to } \\
\text { an 'active category' } \\
\text { ( } f \text { at the time of the } \\
\text { study) ( } f \text { inflated to } \\
\text { 2011) }\end{array}$ & $\begin{array}{l}\text { ICER ( } f \text { at the time of } \\
\text { the study) ( } \mathrm{f} \text { inflated } \\
\text { to 2011) }\end{array}$ \\
\hline $\begin{array}{l}\text { Anokye et al, } \\
\text { UK, good }\end{array}$ & $\begin{array}{l}\text { Brief exercise advice in } \\
\text { primary care; healthcare } \\
\text { (NHS), 2010/2011 }\end{array}$ & $\begin{array}{l}\text { Economic } \\
\text { modelling; CUA }\end{array}$ & $\begin{array}{l}\text { I: brief advice } \\
\text { C: usual care (no active } \\
\text { intervention) }\end{array}$ & $\begin{array}{l}\text { Cohort of } 100000 \\
\text { physically inactive } \\
\text { but healthy adults } \\
\text { aged } \geq 33 \text { years }\end{array}$ & $\begin{array}{l}\text { Modelled for } \\
\text { lifetime }\end{array}$ & $\begin{array}{l}150 \mathrm{~min} \text { of MPA } \\
\text { or } 75 \mathrm{~min} \text { of VPA } \\
\text { per week }\end{array}$ & Not mentioned & $\begin{array}{l}\text { f136 (brief advice } \\
\text { compared with usual } \\
\text { care) }\end{array}$ & f1730/QALY gained \\
\hline $\begin{array}{l}\text { Boehler et } a l_{,}^{36} \\
\text { UK, fair }\end{array}$ & $\begin{array}{l}\text { PA promotion in primary } \\
\text { care; health service } \\
\text { (NHS), } 2007\end{array}$ & $\begin{array}{l}\text { PA care } \\
\text { pathway } \\
\text { pilot-based } \\
\text { regression } \\
\text { model; CEA }\end{array}$ & $\begin{array}{l}\text { Brief exercise comparing } 2 \\
\text { recruitment strategies: } \\
\text { I1: patients recruited } \\
\text { opportunistically } \\
\text { 12: patients on the hypertension } \\
\text { disease register 'disease register } \\
\text { sites' }\end{array}$ & $\begin{array}{l}\text { Insufficiently active, } \\
16-74 \text { years }\end{array}$ & 3 months & $\begin{array}{l}150 \text { min of MPA } \\
\text { per week }\end{array}$ & $\begin{array}{l}\text { 11: } 4 \text { min } \\
\text { 12: } 18 \text { min } \\
\text { Total across } \\
\text { intervention: } \\
\text { I1: } 28 \text { min } \\
\text { 12: } 76 \text { min }\end{array}$ & $\begin{array}{l}\text { f886.50 (f986) disease } \\
\text { register vs } \\
\text { opportunistic } \\
\text { recruitment }\end{array}$ & \\
\hline $\begin{array}{l}\text { Cobiac et al, } \\
\text { Australia, high }\end{array}$ & $\begin{array}{l}\text { PA promotion in } \\
\text { community; health sector, } \\
2003\end{array}$ & $\begin{array}{l}\text { Economic } \\
\text { modelling } \\
\text { study; CUA }\end{array}$ & $\begin{array}{l}\text { I1: GP prescription-exercise } \\
\text { prescription with follow-up } \\
\text { phone call } \\
\text { 12: GP referral for PA counselling } \\
\text { to an exercise physiologist } \\
\text { 13: mass media } \\
\text { 14: TravelSmart (active transport) } \\
\text { 15: pedometer } \\
\text { 16: internet advice } \\
\text { C: do nothing }\end{array}$ & $\begin{array}{l}\text { 11: } 40-79 \text { years old } \\
\text { less active patients } \\
\text { 12: } 60+\text { years } \\
\text { sedentary } \\
\text { 13: } 25-60 \text { years } \\
\text { 14: } 18+\text { years } \\
\text { 15: } 15+\text { years } \\
\text { 16: } 15+\text { years }\end{array}$ & $\begin{array}{l}\text { Modelled for } \\
\text { lifetime }\end{array}$ & $\begin{array}{l}150 \text { min of } \\
\text { moderate } \\
\text { intensity of } 5 \\
\text { METs per week }\end{array}$ & Not mentioned & & $\begin{array}{l}\text { I1: \$A11 } 000 \text { (f5374) } \\
\text { (f6500) per DALY } \\
\text { I2: \$A75 000 (f36 638) } \\
\text { (f44 315) per DALY } \\
\text { I3: dominant } \\
\text { I4: \$A } 18000 \text { (f8793) } \\
\text { (f10 636) per DALY } \\
\text { I5: dominant } \\
\text { I6: \$A2000 (f977) } \\
\text { (f1182) per DALY } \\
\text { when compared with } \\
\text { 'do nothing' }\end{array}$ \\
\hline $\begin{array}{l}\text { Dalziel et al, } \\
\text { New Zealand, } \\
\text { high }\end{array}$ & $\begin{array}{l}\text { Primary care-based } \\
\text { exercise counselling/ } \\
\text { prescription; health } \\
\text { system, } 2001\end{array}$ & $\begin{array}{l}\text { RCT-based } \\
\text { economic } \\
\text { modelling; CUA }\end{array}$ & $\begin{array}{l}\text { I: green prescription } \\
\text { C: usual care (no additional } \\
\text { exercise advice) }\end{array}$ & $\begin{array}{l}\text { Less active } \\
\text { participants; } \\
40-79 \text { years } \\
\text { I: } 451 \\
\text { C: } 427\end{array}$ & $\begin{array}{l}\text { Modelled over } \\
\text { full life } \\
\text { expectancy }\end{array}$ & $\begin{array}{l}5 \times 30 \text { min of } \\
\text { MPA or VPA per } \\
\text { week }\end{array}$ & $\begin{array}{l}7 \text { min (GP); } 13 \\
\text { min (practice } \\
\text { nurse) }\end{array}$ & & $\begin{array}{l}\text { NZD } 2053 \text { (f865) } \\
\text { (f1104) per QALY } \\
\text { (lifetime) }\end{array}$ \\
\hline $\begin{array}{l}\text { Elley et al, }{ }^{42} \\
\text { New Zealand, } \\
\text { high }\end{array}$ & $\begin{array}{l}\text { Primary care exercise } \\
\text { counselling/prescription; } \\
\text { health funder's and } \\
\text { societal, } 2001\end{array}$ & RCT; CEA & $\begin{array}{l}\text { I: green prescription (brief oral or } \\
\text { written advice) by a GP or } \\
\text { practice nurse with telephone } \\
\text { exercise specialist follow-up } \\
\text { C: usual care (do nothing) }\end{array}$ & $\begin{array}{l}40-79 \text { years old less } \\
\text { active patients in } \\
\text { general practice } \\
\text { I: } 451 \\
\text { C: } 427\end{array}$ & 12 months & $150 \mathrm{~min} /$ week & $\begin{array}{l}7 \text { min (GP); } \\
13 \text { min (nurse) }\end{array}$ & $\begin{array}{l}\text { NZD } 1756(£ 740) \\
(\mathrm{f} 938)\end{array}$ & \\
\hline \multirow[t]{2}{*}{$\begin{array}{l}\text { Elley et al, }{ }^{41} \\
\text { New Zealand, } \\
\text { high }\end{array}$} & $\begin{array}{l}\text { Primary care exercise } \\
\text { counselling/prescription } \\
\text { with ongoing support; } \\
\text { Societal, } 2008\end{array}$ & $\mathrm{RCT} ; \mathrm{CEA}$ & $\begin{array}{l}\text { I: enhanced green prescription } \\
\text { (nurse-delivered brief advice and } \\
\text { a written exercise prescription, } \\
\text { counselling in primary care with } \\
\text { telephone follow-up) } \\
\text { C: usual care (do nothing) }\end{array}$ & $\begin{array}{l}\text { Physically inactive } \\
\text { women aged } 40-74 \\
\text { years } \\
\text { I: } 544 \\
\text { C: } 545\end{array}$ & 24 months & $\begin{array}{l}150 \mathrm{~min} / \text { week of } \\
\text { at least MPA }\end{array}$ & $\begin{array}{l}10 \text { min brief } \\
\text { advice and a } \\
\text { written } \\
\text { prescription }\end{array}$ & $\begin{array}{l}\text { NZD } 687 \text { ( } £ 285)(£ 308) \\
\text { sustained at } \\
12 \text { months; NZD } 1407 \\
\text { (f584) (f630) } \\
\text { sustained at } 24 \text { months }\end{array}$ & \\
\hline & $\begin{array}{l}\text { Universal strategy to } \\
\text { promote PA in primary }\end{array}$ & $\begin{array}{l}\text { Economic } \\
\text { modelling; CUA }\end{array}$ & $\begin{array}{l}\text { I: brief GP advice in primary care } \\
\text { C: standard care (do nothing) }\end{array}$ & $\begin{array}{l}262704 \text { healthy } \\
\text { participants aged }\end{array}$ & $\begin{array}{l}\text { Modelled for } \\
\text { lifetime }\end{array}$ & & Not mentioned & & $\begin{array}{l}\text { Net health benefit of } \\
3.2 \text { QALYs per } 1000\end{array}$ \\
\hline
\end{tabular}




\begin{tabular}{|c|c|c|c|c|c|c|c|c|c|}
\hline \multirow[b]{2}{*}{$\begin{array}{l}\text { Study, } \\
\text { setting, } \\
\text { quality }\end{array}$} & \multirow[b]{2}{*}{$\begin{array}{l}\text { Objective; economic } \\
\text { perspective, cost year }\end{array}$} & \multirow[b]{2}{*}{$\begin{array}{l}\text { Study type; } \\
\text { economic } \\
\text { analysis type }\end{array}$} & \multirow[b]{2}{*}{ Interventions compared } & \multirow[b]{2}{*}{ Participants } & \multirow[b]{2}{*}{$\begin{array}{l}\text { Follow-up } \\
\text { duration }\end{array}$} & \multirow[b]{2}{*}{$\begin{array}{l}\text { Definition of } \\
\text { physically } \\
\text { active person }\end{array}$} & \multirow[b]{2}{*}{$\begin{array}{l}\text { Mean time to } \\
\text { deliver } \\
\text { intervention per } \\
\text { person }\end{array}$} & \multicolumn{2}{|l|}{ Outcome } \\
\hline & & & & & & & & $\begin{array}{l}\text { Cost of converting to } \\
\text { an 'active category' } \\
\text { ( } f \text { at the time of the } \\
\text { study) (f inflated to } \\
\text { 2011) }\end{array}$ & $\begin{array}{l}\text { ICER ( } f \text { at the time of } \\
\text { the study) ( } \mathrm{f} \text { inflated } \\
\text { to 2011) }\end{array}$ \\
\hline $\begin{array}{l}\text { Gulliford } \\
\text { et al, } \\
\text { good }\end{array}$ & $\begin{array}{l}\text { care; healthcare service, } \\
2010\end{array}$ & & & $\begin{array}{l}30-100 \text { years from } \\
\text { GPRD }\end{array}$ & & $\begin{array}{l}150 \text { min of } \\
\text { moderate PA per } \\
\text { week }\end{array}$ & & & $\begin{array}{l}\text { participants (at a } \\
\text { threshold of } f 30000 / \\
\text { QALY); } £ 13686 \\
\text { (f14 002)/QALY }\end{array}$ \\
\hline $\begin{array}{l}\text { Leung et } \mathrm{al}_{1}^{43} \\
\text { New Zealand, } \\
\text { High }\end{array}$ & $\begin{array}{l}\text { Pedometer-based exercise } \\
\text { advice to increase PA; } \\
\text { societal, } 2008\end{array}$ & RCT; CUA & $\begin{array}{l}\text { 11: pedometer-based green } \\
\text { prescription } \\
\text { 12: standard green prescription } \\
\text { (exercise advice \& time-related } \\
\text { goal) }\end{array}$ & $\begin{array}{l}\text { Healthy inactive } \\
\text { adults aged } \\
\geq 65 \text { years } \\
\text { I1: } 165 \\
\text { I2: } 165\end{array}$ & 12 months & $\begin{array}{l}150 \text { min of at } \\
\text { least MPA per } \\
\text { week }\end{array}$ & Not mentioned & $\$ A 667(\mathrm{f} 290)(\mathrm{f} 313)$ & \\
\hline $\begin{array}{l}\text { Lindgren } \\
\text { et al,46 } \\
\text { Sweden, good }\end{array}$ & $\begin{array}{l}\text { Dietary and exercise } \\
\text { advice; societal and } \\
\text { payer's, } 2000\end{array}$ & $\begin{array}{l}\text { RCT-based } \\
\text { economic } \\
\text { modelling; CEA }\end{array}$ & $\begin{array}{l}\text { 11: dietary advice by dieticians } \\
\text { 12: exercise advice by a physician } \\
\text { I3: exercise \& diet } \\
\text { C: usual care (no intervention) }\end{array}$ & $\begin{array}{l}\text { Men aged } \\
35-60 \text { years } \\
\text { I1: } 40 \\
\text { I2: } 39 \\
\text { I3: } 39 \\
\text { C: } 39\end{array}$ & $\begin{array}{l}\text { Modelled for } \\
\text { lifetime }\end{array}$ & $\begin{array}{l}\text { Regular PA of an } \\
\text { aerobic type 2-3 } \\
\text { times/week } \\
\text { lasting 30- } \\
45 \text { min }\end{array}$ & $\begin{array}{l}\text { Not mentioned } \\
\text { but included } 3 \\
\text { visits to a } \\
\text { physician }\end{array}$ & & $\begin{array}{l}\text { SEK } 180470 \text { (f12 263) } \\
\text { ( } \mathrm{f15} 873 \text { ) per LYG for } \\
\text { exercise compared to } \\
\text { no intervention }\end{array}$ \\
\hline $\begin{array}{l}\text { Over et al, } \\
\text { Netherlands, } \\
\text { good }\end{array}$ & $\begin{array}{l}\text { GP counselling in } \\
\text { addition to pedometers } \\
\text { to increase PA; } \\
\text { healthcare, } 2009\end{array}$ & $\begin{array}{l}\text { Economic } \\
\text { modelling; CUA }\end{array}$ & $\begin{array}{l}\text { Two scenarios } \\
\text { S1: pedometer use with diary } \\
\text { and GP counselling } \\
\text { S2: current practice (no } \\
\text { additional advice) }\end{array}$ & $\begin{array}{l}\text { Insufficiently active } \\
20-65 \text { years }\end{array}$ & $\begin{array}{l}\text { Modelled for } \\
\text { lifetime }\end{array}$ & $\begin{array}{l}150 \text { min of MPA } \\
\text { per week }\end{array}$ & $\begin{array}{l}\text { Not mentioned } \\
\text { but included } \\
10 \text { min GP } \\
\text { counselling }\end{array}$ & & $\begin{array}{l}€ 11100 \text { (f8401) } \\
\text { (f8858) per QALY }\end{array}$ \\
\hline $\begin{array}{l}\text { Pringle et } a l_{,}^{38} \\
\text { UK, fair }\end{array}$ & $\begin{array}{l}\text { Community-based } \\
\text { interventions to increase } \\
\text { MPA; NHS, } 2003\end{array}$ & $\begin{array}{l}\text { Alongside } \\
\text { single clinical } \\
\text { and cost study; } \\
\text { CEA, CUA }\end{array}$ & $\begin{array}{l}\text { Seven intervention categories: } \\
\text { campaigns, exercise classes, } \\
\text { exercise referral, motivational } \\
\text { interviews, outdoor activity, } \\
\text { peer-mentoring, training of PA } \\
\text { leaders }\end{array}$ & $\begin{array}{l}\text { Inactive; } 343 \text { young } \\
\text { people and } 641 \\
\text { adults, particularly } \\
\text { those aged } 65 \text { years } \\
\text { and over }\end{array}$ & $\begin{array}{l}\text { Modelled for } \\
\text { lifetime using } \\
\text { the Matrix } \\
\text { model }^{16}\end{array}$ & $\begin{array}{l}150 \text { min of MPA } \\
\text { per week }\end{array}$ & Not mentioned & $\begin{array}{l}\mathrm{f} 260-\mathrm{f} 1253 \\
\text { (f318-f1531) per } \\
\text { completer improving } \\
\text { MPA }\end{array}$ & $\begin{array}{l}f 47-f 229(f 57-f 280) \\
\text { per QALY }\end{array}$ \\
\hline $\begin{array}{l}\text { Shaw et al, } \\
\text { Scotland, fair }\end{array}$ & $\begin{array}{l}\text { Pedometer-based } \\
\text { walking; health services, } \\
2008\end{array}$ & RCT; CEA & $\begin{array}{l}\text { I: minimal intervention (walking } \\
\text { programme and pedometer) } \\
\text { I2: maximal intervention (PA } \\
\text { consultation, pedometer and } \\
\text { individualised walking } \\
\text { programme) } \\
\text { C: 'usual behaviour' }\end{array}$ & $\begin{array}{l}\text { 18-65 years olds } \\
\text { 11: } 40 \\
\text { I2: } 39\end{array}$ & 12 months & $\begin{array}{l}\text { Weekly increase } \\
\text { of } \geq 15000 \text { steps }\end{array}$ & $30 \mathrm{~min}$ & $\begin{array}{l}\mathrm{f} 92 \text { (f99) (minimal vs } \\
\text { control) } \\
\text { f591 (f637) (maximal } \\
\text { vs minimal) }\end{array}$ & \\
\hline $\begin{array}{l}\text { Sims et al, }{ }^{45} \\
\text { Australia, fair }\end{array}$ & $\begin{array}{l}\text { Active script in general } \\
\text { practice; health service, } \\
1996\end{array}$ & $\begin{array}{l}\text { Economic } \\
\text { modelling; CEA, } \\
\text { CUA }\end{array}$ & $\begin{array}{l}\text { I: ASP_improving systematic PA } \\
\text { advice by GPs } \\
\text { C: routine GP care (no PA advice) }\end{array}$ & $\begin{array}{l}\text { Less active adults } \\
\text { aged } 20-75 \text { years, } \\
670 \mathrm{GP} \text { advising } \\
\text { sedentary patients } \\
\text { I: } 40258 \\
\text { C: } 10437\end{array}$ & $\begin{array}{l}\text { Unclear time } \\
\text { horizon }\end{array}$ & $\begin{array}{l}150 \text { min of MPA } \\
\text { per week }\end{array}$ & $\begin{array}{l}4 \text { min GP } \\
\text { consultation }\end{array}$ & $\begin{array}{l}\text { \$138 (f70) ( }(996) \text { per } \\
\text { patient to become } \\
\text { active }\end{array}$ & $\begin{array}{l}\text { \$A3647 (f1838) } \\
\text { (f2542) per DALY } \\
\text { saved }\end{array}$ \\
\hline
\end{tabular}

ASP, Active Script Programme; CEA, cost-effectiveness analysis; CUA, cost-utility analysis; DALY, disability adjusted life year; GP, general practitioner; GPRD, general practice research database; ICER, incremental cost-effectiveness ratio; LYG, life years

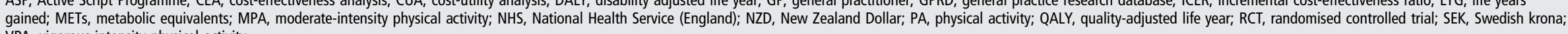
VPA, vigorous-intensity physical activity. 


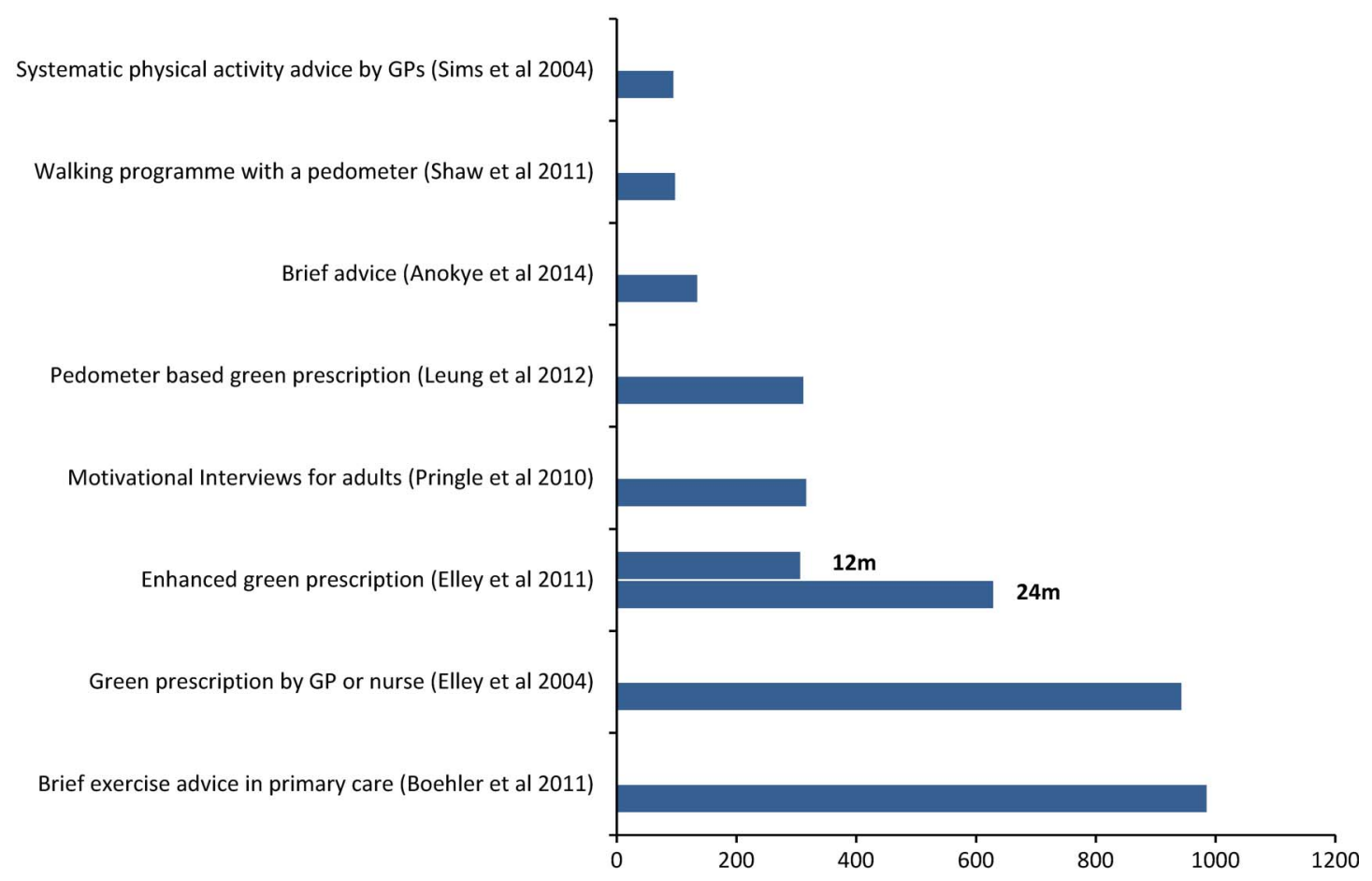

Figure 2 Incremental cost of converting one sedentary adult to an active category (2011 equivalent $f$ sterling; GP, general practitioner).

recruitment). They reported the incremental cost of converting one inactive adult to an active state as $£ 986$ in disease register screening compared to opportunistic screening. Elley et $a l^{41}$ reported the cost-effectiveness of 'enhanced green prescription' (nurse delivered exercise counselling and written prescription with telephone follow-up) at both 12 and 24 months postintervention, which showed decreasing cost-effectiveness over time of follow-up ( $£ 308$ at 12 months vs $£ 630$ at 24 months).

\section{Studies reporting final outcomes}

Figure 3 summarises the results in terms of incremental cost utility, that is, per QALY or DALY or LYG. Pedometer-based brief interventions appeared likely to yield health gains at a lower cost. Pedometers (either as a motivational tool or in combination with exercise advice) were dominant as they were both cost saving and more effective when compared with usual care ${ }^{44}$ or standard 'green prescription' (oral or written exercise advice by a GP or practice nurse with telephone follow-up). ${ }^{43}$ GP counselling in combination with a pedometer when compared with current practice had a cost-utility of $£ 8858 /$ QALY. $^{47}$

Pringle $e t a l^{38}$ evaluated the costs and cost-effectiveness of seven broad categories of community-based interventions. Among these, only motivational interviews are relevant to the scope of this review. The analysis was conducted alongside a pre-intervention and post-intervention design and hence no 'usual care' or 'do nothing' control group was considered. Cobiac et $\mathrm{al}^{44}$ compared six intervention strategies, each against 'do nothing', of which pedometers and exercise on prescription are relevant to this review. They reported that the use of pedometers was more cost-effective than other interventions. Likewise, Lindgren et $a l^{46}$ modelled the cost utility of three interventions of dietary and exercise advice of which exercise advice by a physician was relevant to this review. The estimated cost utility of exercise advice by a physician was $£ 15873 / \mathrm{LYG}$ when compared with usual care.

The incremental cost-effectiveness ratio (ICER) for exercise advice or exercise on prescription (vs usual care) ranged from
$£ 1104$ to $£ 14002 / \mathrm{QALY}^{35} 37{ }^{40}$ and from $£ 2542$ to $£ 6500 /$ DALY. $^{44}{ }^{45}$ Motivational interviews had the lowest ICER ( $£ 57$ / QALY). ${ }^{38}$ In a primary care setting, GP counselling in combination with a pedometer ${ }^{47}$ had a lower cost-effectiveness ratio than a GP's advice or counselling with written materials ${ }^{37}$ ( $£ 8858$ vs $£ 14002 / \mathrm{QALY}$ ); both were compared with usual care.

These economic modelling studies used either a multistate life-table modelling approach ${ }^{44}$ or Markov chain ${ }^{35} 374647$ or a decision tree. ${ }^{38}$ Effectiveness data used in these modelling studies had a follow-up duration of up to 2 years.

\section{DISCUSSION}

The studies identified in this review suggest that brief interventions can result in a meaningful increase in physical activity at reasonable costs. When the longer term costs and health benefits are considered, brief interventions are cost-effective compared with the NICE threshold of $£ 20000-£ 30000$ /QALY gained.

\section{Successful interventions-'bright spots'}

Exercise advice, ${ }^{35} 45$ pedometer-based walking, ${ }^{39}$ pedometerbased 'green prescription ${ }^{, 43}$ and motivational interviews ${ }^{38}$ had similar cost-effectiveness ratios for converting one inactive person to an active state. The 'active script programme' evaluated by Sims et $a l^{45}$ had the lowest cost-effectiveness in terms of cost per additional active person. However, their economic model had rather optimistic assumptions regarding the uptake of physical activity by intervention and control group patients, and the time horizon of the study was not clear. From the reviewed studies, we observed that in the absence of continued contacts, some of the gains in physical activity experienced by participants are likely to be lost over time, resulting in the intervention becoming less cost-effective over a longer time horizon, as was evident in the analysis of 'enhanced green prescription'. ${ }^{41}$

Promoting pedometer use, ${ }^{44}$ pedometer in combination with exercise on prescription, ${ }^{43}$ motivational interviews, ${ }^{38} \mathrm{GP}$ advice or counselling on exercise ${ }^{4045}$ and brief advice ${ }^{35}$ were the most cost-effective intervention strategies with respect to cost utility. 


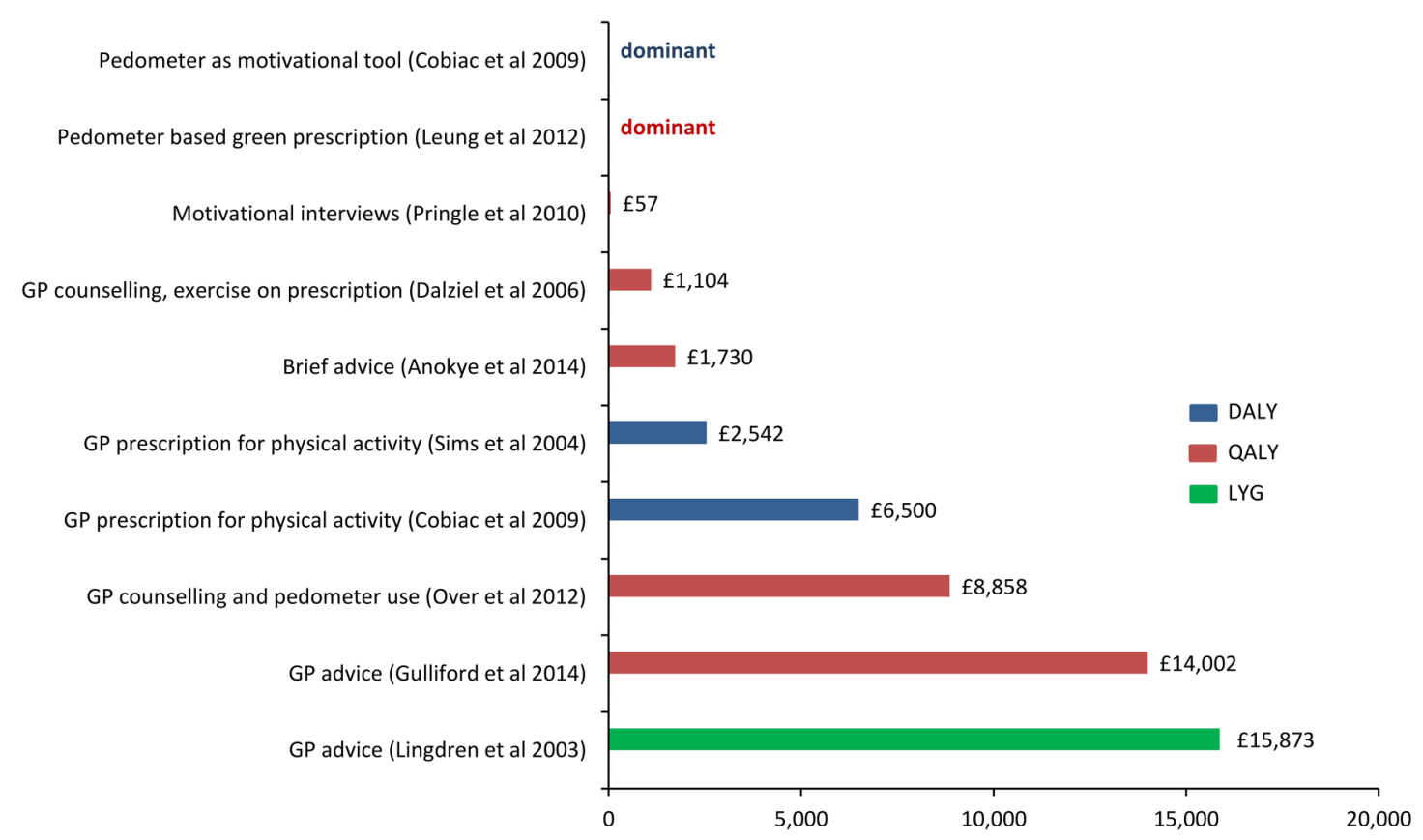

Figure 3 Cost-effectiveness ratios (cost per DALY or QALY or LYG) for different physical activity interventions (2011 equivalent $f$ sterling; DALY, disability-adjusted life year; GP, general practitioner; LYG, life years gained; QALY, quality-adjusted life year).

Both Leung et $a l^{43}$ and Cobiac et $a l^{44}$ found the costeffectiveness of their pedometer intervention to be dominant, indicating that the pedometer intervention is more effective and less costly than comparators. However, note that Leung et al ${ }^{43}$ compared pedometer-based 'green prescription' with standard 'green prescription' rather than with usual care. In contrast, Over et $a l^{47}$ reported a considerably higher ICER for pedometer intervention compared to current practice ( $£ 8858 / \mathrm{QALY}$ ). Although the effectiveness data for both the Cobiac et $a l^{44}$ and Over et $a l^{47}$ studies were based on a meta-analysis by Bravata et $a l,{ }^{63}$ the higher possible health gains in Cobiac et al ${ }^{44}$ may be a consequence of the much larger proportion of inactive people in the Australian population compared to the Dutch population, and of the reported programme cost per participant being lower in the Australian estimates than in the Over et al studies. ${ }^{47}$

Anokye $e t a l^{35}$ modelled the cost-effectiveness of brief advice in primary care using meta-analysed data, but the underlying primary evidence included both RCTs and non-randomised studies. A primary care nurse delivered 'enhanced green prescription ${ }^{41}$ had a more favourable ICER than a GP delivered 'green prescription'42 ( $£ 308$ vs $£ 938$ for converting one additional inactive adult to an active state over a 12 -month period). The 'enhanced green prescription' had a slightly higher proportion of people who increased their activity at 12 months than just 'green prescription', which was most likely attributable to the extra telephone support and 6-month face-to-face, nurse-led follow-up. Three studies 404244 evaluated the cost-effectiveness of 'green prescription' using data from a cluster RCT. ${ }^{20}$ However, they reported different cost outcomes-cost per DALY, $^{44}$ QALY $^{40}$ or the intermediate outcome. ${ }^{42}$

Some of the modelling studies adopted previously reported economic models. For example, Pringle et al's ${ }^{38}$ model was informed by the NICE cost-effectiveness model, ${ }^{16}$ while Over et $a l^{47}$ used the Dutch National Institute for Public Health and the Environment (RIVM) Chronic Disease Model to estimate the long-term effects of increase in physical activity. The particular disease states included in a model affect the long-term estimates of cost and health outcome. Lindgren et $a l^{46}$ included only CHD conditions in their model while others included multiple conditions (type 2 diabetes, CHD, breast and colorectal cancers and depression).

\section{Methodological issues to take into account}

Some of the studies failed to report sources for unit costs, and some potentially relevant costs were being excluded from the analysis. For example, Dalziel et $a l^{40}$ included only programme costs, but excluded the healthcare cost implications of downstream health events. Similarly, Pringle et $a l^{38}$ did not include out-of-pocket expenditures which might be significant and could influence the intervention attendance levels (apart from the cost-effectiveness of the intervention).

The model-based economic evaluations of brief interventions differ mainly in terms of the quality of evidence used, structure and outcome measure. Use of data on the effect of interventions in economic modelling using a systematic review and meta-analysis of RCTs is proposed as the least biased source of data. ${ }^{64}$ LYG is a pure measurement of life expectancy whereas DALYs and QALYs adjust life expectancy for morbidity, using disability and quality-of-life weighting, respectively. ${ }^{65} 66$ The ICERs for both QALY and DALY outcomes varied between studies-primary care-based exercise advice evaluated by Dalziel et $a l^{40}$ had a lower cost utility ( $£ 1104 /$ QALY) than Gulliford et $a$ ' $^{37}$ study ( $£ 14002 / \mathrm{QALY}$ ). Gulliford $e t a l^{37}$ used effectiveness data from a recent systematic review and meta-analysis of $\mathrm{RCTs}^{23}$ and evaluated two scenarios of universal strategy to promote physical activity. Their model was more comprehensive than Dalziel et al's ${ }^{40}$ model in terms of inclusion of disease conditions (16 single disease or multi-disease states), model structure and intervention effects.

\section{Sensitivity analysis}

Eight studies included in this review ${ }^{35-37} 4043444647$ properly characterised decision uncertainty by using probabilistic sensitivity analysis while the remaining studies used scenario-based or one-way sensitivity analysis. Four studies ${ }^{39} 41-43$ were based on 'piggybacked' economic evaluations conducted alongside 
rigorous RCTs. These provide a source of evidence on resource use and health effects from well-designed studies with high internal validity. However, they are often constrained in terms of the range of outcome data collected or the length of follow-up, introducing difficulties in extrapolating the intervention effectiveness beyond the trial period. ${ }^{67}$ For example, the assumptions around the maintenance of physical activity levels beyond brief interventions determine how cost-effective the intervention is. In addition, it should be recognised that economic analysis in three studies ${ }^{36} 3845$ was based on evidence from non-experimental studies or theoretical scenarios.

\section{Comparison with previous reviews}

Three previous economic reviews of physical activity interventions were identified. A recent systematic review commissioned by $\mathrm{NICE}^{29}$ reported that brief advice in primary care was costeffective (when usual care was used as the benchmark). However, the evidence was limited to three studies. Garrett $e t a l^{28}$ reviewed the cost-effectiveness of physical activity interventions in primary care and the community, and included 13 RCTs. The review also included intensive interventions but did not include modelling studies. They reported that most interventions were costeffective, especially where direct supervision or instruction was not required. The cost-effectiveness ratios for moving one inactive person to an active stage at 12 months varied from $£ 262$ to $£ 3144$, and the cost-utility estimated in nine studies varied from $£ 276$ to $£ 68798$ /QALY gained (2011 prices). Another review by Muller-Riemenschneide $e t{ }^{25}$ included eight studies (6 RCTs, 1 cross-sectional and 1 economic modelling) covering a broad range of interventions promoting physical activity. The review concluded that behavioural interventions targeting sedentary healthy adults can achieve the recommended level of physical activity at a cost of $£ 662$ per participant over a 12 -month period (2011 prices). However, their review included workplacebased physical activity and environmental interventions.
These reviews either considered the cost-effectiveness of physical activity interventions in general and were not specific to brief interventions ${ }^{25} 28$ or did not include other kinds of brief interventions for physical activity (eg, pedometer-based interventions). ${ }^{29}$ Although six studies included in our review were also included in the previous reviews (figure 4), we include seven additional studies and looked specifically at the costeffectiveness of brief interventions promoting physical activity in the general population.

\section{Strengths and limitations of the review}

To the best of our knowledge, this is the most comprehensive economic review to date of brief interventions for physical activity. We assessed the efficiency of brief interventions in terms of cost-effectiveness ratios, and report both intermediate (ie, incremental cost of converting one inactive adult to an active category) and final outcomes (cost per DALY or QALY or LYG). The current review includes both economic evaluations alongside RCTs and economic modelling, summarising the results of economic evaluation studies of brief interventions in primary care and community settings.

In general, the assessment of the cost-effectiveness of different brief interventions was challenging. They vary widely in terms of the methodology used in their cost-effectiveness or cost-utility analyses, including the perspective of the economic analysis, and the discounting of future values of cost and health outcomes. The reviewed modelling studies have their own limitations: the assumptions underlying the models can differ considerably, for instance, as regards the assumptions on the proportion of people becoming active as a result of the brief intervention, outcome measures and on the decay in intervention effect over time. Such methodological differences between the studies as well as other context characteristics (eg, the variability in funding mechanism, health system and cost structures) limit the generalisability of the cost-effectiveness results across
Figure 4 Venn diagram showing overlap of studies with previous reviews.

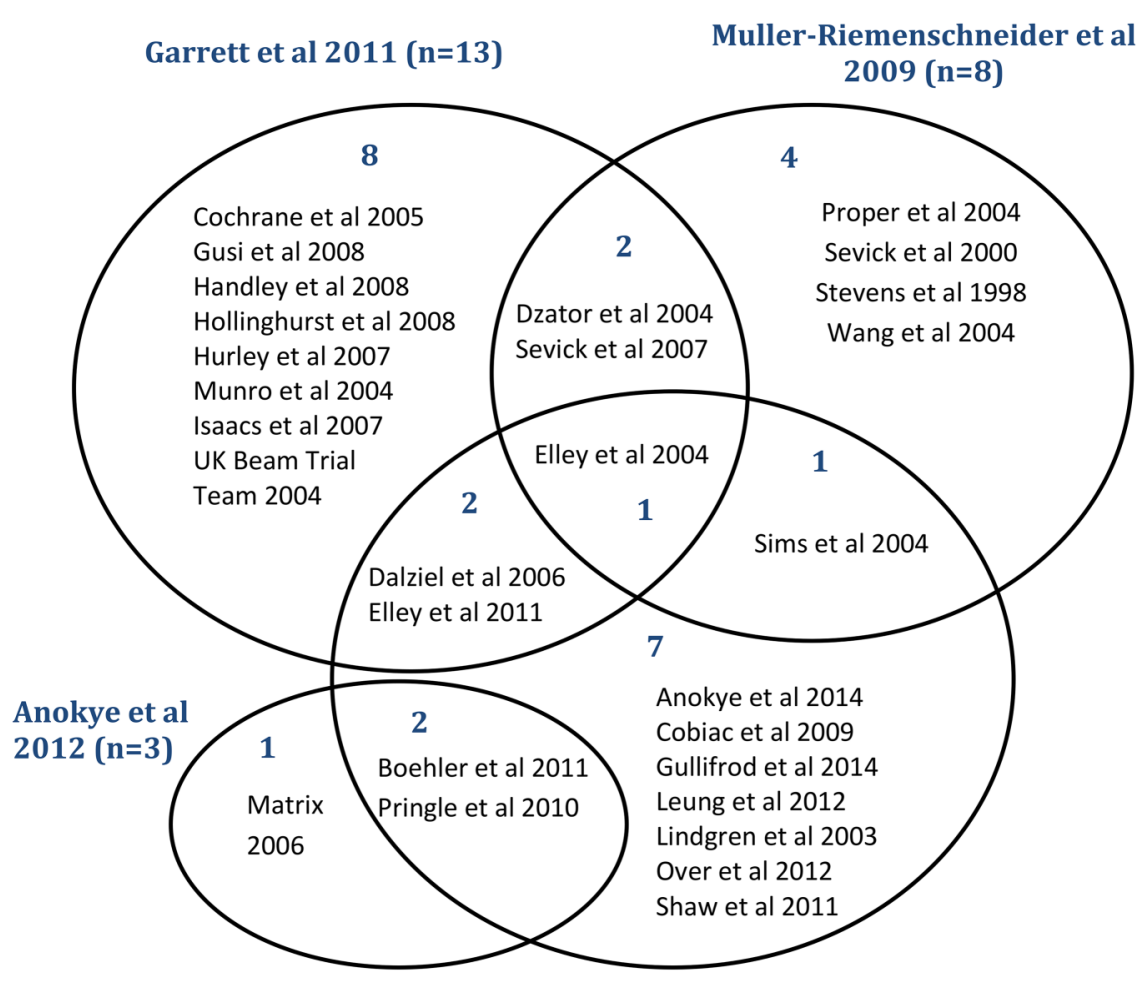

Current review $(n=13)$ 
different settings. ${ }^{68}$ In addition, some of the studies included in this analysis lack intervention details, for example, time duration and/or delivery method (eg, type of provider and individual vs group delivery), making it difficult to determine whether or not the interventions were truly 'brief interventions' according to the NICE definition. It is important to describe interventions in sufficient detail, ${ }^{69}$ such as the duration of 'brief interventions', as it affects cost-effectiveness. In this review, we included brief physical activity interventions that had at least one (initial) face-to-face contact and acknowledge that there is a need for economic reviews of non-face-to-face physical activity interventions which constitute a fast growing research area.

It would be more appropriate to compare each intervention in an iterative manner taking an account of dominance and extended dominance to determine the most cost-effective intervention. ${ }^{70}$ The interventions included in this review were typically compared with a usual care (do nothing) intervention that compromises the ability to rank interventions. What is required in order to make this comparison is a single framework (ie, decision analytic model) to transform the short-term costs and intermediate ('disease specific') outcomes into longer term final outcomes (namely QALYs), in order both to identify the most cost-effective intervention strategy and to quantify the associated decision uncertainty.

\section{Summary}

Brief interventions delivered at the individual level by a GP, practice nurse or other healthcare professional can increase physical activity in healthy inactive adults at a reasonable cost to convert one inactive adult to being 'active'. Our findings suggest that it is possible to deliver a brief intervention for less than $£ 15$ 000/QALY gained. On the basis of the cost-utility analysis, the use of pedometers and motivational interviews had the lowest cost-effectiveness ratio. Heterogeneity in interventions, study participants and study design compromises the comparability of the results across studies. It is thus difficult to rank and prioritise intervention strategies based on cost-effectiveness ratios, though most of these interventions are considered costeffective when measured against the current NICE threshold of

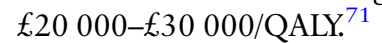

We conclude that brief interventions are likely to be inexpensive, but we have limited knowledge on the longer term costs and consequences of these interventions. ${ }^{30}$ Thus, economic modelling from studies with a longer follow-up will improve estimates

\section{What is already known on this topic}

Brief interventions for physical activity in primary care or community settings are considered to be effective in increasing individuals' physical activity. However, the evidence on the cost-effectiveness of such interventions is sparse.

\section{What this study adds}

Some brief interventions increase individuals' physical activity at reasonable costs and are cost-effective, given commonly accepted thresholds. An economic evaluation of brief interventions with a longer follow-up will improve the estimates of the longer term costs and benefits of physical activity. of the longer term costs and consequences of brief interventions for physical activity in primary care or community settings.

Twitter Follow Ed Wilson at @edcfwilson

Acknowledgements The authors would like to thank Dr Dan Mason and Ms Laura Lamming for their comments on the initial version of this manuscript, and the author(s) of studies included in our review who responded to requests for clarification or further information about study data. This study was conducted on behalf of the Very Brief Interventions Programme team (see http://www.phpc.cam.ac. uk/pcu/research/research-projects-list/vbi/vbi-research-team for team members).

Contributors EW and VGC contributed to the design of the review. VGC executed the search strategy with inputs from EW and MS, reviewed the references found, assessed studies for inclusion, appraised and extracted the data from each of the included studies, and also performed the analysis and prepared the first draft. EW double checked the extracted data for the included studies. EW, MS, SS and WH provided critical comments on quality assessment and data analysis. All authors contributed to the critical revision of the manuscript and approved the final version of the manuscript.

Funding EW is funded by the NIHR Cambridge Biomedical Research Centre. This paper presents independent research funded by the National Institute for Health Research (NIHR) under its Programme Grants for Applied Research Programme (Grant Reference Number RP-PG-0608-10079). The views expressed are those of the author (s) and not necessarily those of the NHS, the NIHR or the Department of Health. The funder had no role in study design, data collection, data analysis, data interpretation, the writing of the manuscript, and decision to submit the manuscript for publication.

\section{Competing interests None declared.}

Provenance and peer review Not commissioned; externally peer reviewed.

Open Access This is an Open Access article distributed in accordance with the Creative Commons Attribution Non Commercial (CC BY-NC 4.0) license, which permits others to distribute, remix, adapt, build upon this work non-commercially, and license their derivative works on different terms, provided the original work is properly cited and the use is non-commercial. See: http://creativecommons.org/ licenses/by-nc/4.0/

\section{REFERENCES}

1 World Health Organization. Global health risks: mortality and burden of disease attributable to selected major risks. Geneva: World Health Organization, 2009.

2 Waxman A. WHO global strategy on diet, physical activity and health. Food Nutr Bull 2004;25:292-302.

3 Oldridge NB. Economic burden of physical inactivity: healthcare costs associated with cardiovascular disease. Eur J Cardiovasc Prev Rehabil 2008;15:130-9.

4 Scarborough P, Bhatnagar P, Wickramasinghe KK, et al. The economic burden of ill health due to diet, physical inactivity, smoking, alcohol and obesity in the UK: an update to 2006-07 NHS costs. J Public Health (Oxf) 2011;33:527-35.

5 Khan KM, Thompson AM, Blair SN, et al. Sport and exercise as contributors to the health of nations. Lancet 2012;380:59-64.

6 Department of Health. At least five a week: evidence on the impact of physical activity and its relationship with health. A report from the Chief Medical Officer. London: Department of Health, 2004.

7 Craig R, Hirani V, eds. Health Survey for England 2009. London: The NHS Information Centre for Health and Social Care, 2010.

8 Hillsdon M, Foster C, Thorogood M. Interventions for promoting physical activity. Cochrane Database Syst Rev 2005;(1):CD003180.

9 Department of Health. Start active, stay active: a report on physical activity from the four home countries' Chief Medical Officers. Department of Health, 2011.

10 Roth M. Self-reported physical activity in adults. In: Craig R, Mindell J, Hirani V, eds. Health Survey for England 2008. Volume 1: physical activity and fitness. Leeds: The NHS Information Centre, 2009:21-58.

11 Scholes S, Mindell J. Physical activity in adults. Chapter 2. In: Craig R, Mindell J, eds. Health Survey for England 2012: health, social care and lifestyles. Leeds: Health and Social Care Information Centre, 2013. http://www.hscic.gov.uk/ catalogue/PUB13218/HSE2012-Ch2-Phys-act-adults.pdf

12 Department of Health. Healthy lives, healthy people: our strategy for public health in England. London: The Stationery Office (Great Britain), 2010.

13 Kahn EB, Ramsey LT, Brownson RC, et al. The effectiveness of interventions to increase physical activity. A systematic review. Am J Prev Med 2002;22:73-107.

14 Muller-Riemenschneider $F$, Reinhold $T$, Nocon $M$, et al. Long-term effectiveness of interventions promoting physical activity: a systematic review. Prev Med 2008;47:354-68.

15 National Institute for Health and Clinical Excellence. Four commonly used methods to increase physical activity: brief interventions in primary care, exercise referral schemes, pedometers and community-based exercise programmes for walking and cycling. Public Health Intervention Guidance No 2. London: National Institute for Health and Clinical Excellence, 2006. 
16 Matrix Research and Consultancy. Modelling the cost effectiveness of physical activity interventions. London: Matrix Research and Consultancy, 2006.

17 National Institute for Health and Clinical Excellence. Walking and cycling: local measures to promote walking and cycling as forms of travel or recreation. NICE public health guidance 41. London: National Institute for Health and Clinical Excellence, 2012

18 National Institute for Health and Care Excellence. Physical activity: brief advice for adults in primary care. London: NICE, May 2013.

19 National Institute for Health and Care Excellence. Exercise referral schemes to promote physical activity. London: NICE, September 2014.

20 Elley CR, Kerse N, Arroll B, et al. Effectiveness of counselling patients on physical activity in general practice: cluster randomised controlled trial. BMJ 2003:326:793.

21 Swinburn BA, Walter LG, Arroll B, et al. The green prescription study: a randomized controlled trial of written exercise advice provided by general practitioners. Am J Public Health 1998;88:288-91.

22 Lawton BA, Rose SB, Elley CR, et al. Exercise on prescription for women aged 4074 recruited through primary care: two year randomised controlled trial. BMJ 2008;337:a2509

23 Orrow G, Kinmonth AL, Sanderson S, et al. Effectiveness of physical activity promotion based in primary care: systematic review and meta-analysis of randomised controlled trials. BMJ 2012;344:e1389.

24 Campbell F, Blank L, Messina J, et al. National Institute for Health and Clinical Excellence (NICE) public health intervention guidance physical activity: brief advice for adults in primary care. Review of effectiveness evidence. London: NICE, 2012.

25 Muller-Riemenschneider F, Reinhold T, Willich SN. Cost-effectiveness of interventions promoting physical activity. Br J Sports Med 2009;43:70-6.

26 Gordon L, Graves N, Hawkes A, et al. A review of the cost-effectiveness of face-to-face behavioural interventions for smoking, physical activity, diet and alcohol. Chronic IIIn 2007;3:101-29.

27 Zheng $\mathrm{H}$, Ehrlich $\mathrm{F}$, Amin J. Economic evaluation of the direct healthcare cost savings resulting from the use of walking interventions to prevent coronary heart disease in Australia. Int J Health Care Finance Econ 2010;10:187-201.

28 Garrett S, Elley CR, Rose SB, et al. Are physical activity interventions in primary care and the community cost-effective? A systematic review of the evidence. $\mathrm{Br} J \mathrm{Gen}$ Pract 2011;61:e125-133.

29 Anokye N, Jones T, Fox-Rushby J. National Institute for Health and Clinical Excellence Public Health Intervention Guidance Physical activity-brief advice for adults in primary care: economic analysis. Review of economic evidence. London: NICE, 2012.

30 Davis JC, Verhagen E, Bryan S, et al. 2014 consensus statement from the first Economics of Physical Inactivity Consensus (EPIC) conference (Vancouver). Br J Sports Med 2014;48:947-51.

31 West D, Saffin K. Literature review: brief interventions and childhood obesity. North West and London Teaching Public Health Networks, June 2008.

32 Drummond MF, Sculpher MJ, Torrance GW, et al. Methods for the economic evaluation of health care programmes. Oxford: Oxford University Press, 2005.

33 Shemilt I, Thomas J, Morciano M. A web-based tool for adjusting costs to a specific target currency and price year. Evid Policy 2010;6:51-9.

34 The Campbell and Cochrane Economics Methods Group (CCEMG) and the Evidence for Policy and Practice Information and Coordinating Centre (EPPI-Centre). CCEMG -EPPI-Centre Cost Converter (v1.4): The Campbell and Cochrane Economics Methods Group (CCEMG) and the Evidence for Policy and Practice Information and Coordinating Centre (EPPI-Centre). 2014 (12 Sep 2014). http://eppi.ioe.ac.uk/ costconversion/default.aspx

35 Anokye NK, Lord J, Fox-Rushby J. Is brief advice in primary care a cost-effective way to promote physical activity? Br J Sports Med 2014;48:202-6.

36 Boehler $\mathrm{CE}$, Milton $\mathrm{KE}$, Bull FC, et al. The cost of changing physical activity behaviour: evidence from a "physical activity pathway" in the primary care setting. BMC Public Health 2011;11:370.

37 Gulliford MC, Charlton J, Bhattarai N, et al. Impact and cost-effectiveness of a universal strategy to promote physical activity in primary care: population-based cohort study and Markov model. Eur J Health Econ 2014;15:341-51.

38 Pringle A, Cooke C, Gilson N, et al. Cost-effectiveness of interventions to improve moderate physical activity: a study in nine UK sites. Health Educ $J$ 2010;69:211-24.

39 Shaw R, Fenwick E, Baker G, et al. 'Pedometers cost buttons': the feasibility of implementing a pedometer based walking programme within the community. BMC Public Health 2011;11:200.

40 Dalziel K, Segal L, Elley CR. Cost utility analysis of physical activity counselling in general practice. Aust N Z J Public Health 2006;30:57-63.

41 Elley CR, Garrett S, Rose SB, et al. Cost-effectiveness of exercise on prescription with telephone support among women in general practice over 2 years. $\mathrm{Br} J$ Sports Med 2011:45:1223-9.

42 Elley R, Kerse N, Arroll B, et al. Cost-effectiveness of physical activity counselling in general practice. N Z Med J 2004;117:U1216.

43 Leung W, Ashton T, Kolt GS, et al. Cost-effectiveness of pedometer-based versus time-based Green Prescriptions: the Healthy Steps Study. Aust I Prim Health 2012;18:204-11.
44 Cobiac LJ, Vos T, Barendregt JJ. Cost-effectiveness of interventions to promote physical activity: a modelling study. PLoS Med 2009;6:e1000110.

45 Sims J, Huang N, Pietsch J, et al. The Victorian Active Script Programme: promising signs for general practitioners, population health, and the promotion of physical activity. Br J Sports Med 2004;38:19-25

46 Lindgren $\mathrm{P}$, Fahlstadius $\mathrm{P}$, Hellenius $\mathrm{ML}$, et al. Cost-effectiveness of primary prevention of coronary heart disease through risk factor intervention in 60-year-old men from the county of Stockholm - a stochastic model of exercise and dietary advice. Prev Med 2003;36:403-9.

47 Over EA, Wendel-Vos GW, van den Berg M, et al. Cost-effectiveness of counseling and pedometer use to increase physical activity in the Netherlands: a modeling study. Cost Eff Resour Alloc 2012;10:13.

48 Munro JF, Nicholl JP, Brazier JE, et al. Cost effectiveness of a community based exercise programme in over 65-year-olds: cluster randomised trial. J Epidemiol Community Health 2004; 58:1004-10.

49 Rome A, Persson U, Ekdahl C, et al. Physical activity on prescription (PAP): costs and consequences of a randomized, controlled trial in primary healthcare. Scand J Prim Health Care 2009;27:216-22.

50 Hatziandreu El, Koplan JP, Weinstein MC, et al. A cost-effectiveness analysis of exercise as a health promotion activity. Am J Public Health 1988;78:1417-21.

51 Eriksson MK, Hagberg L, Lindholm L, et al. Quality of life and cost-effectiveness of a 3-year trial of lifestyle intervention in primary health care. Arch Intern Med 2010;170:1470-9.

52 Goyder E, Hind D, Breckon J, et al. A randomised controlled trial and cost-effectiveness evaluation of 'booster' interventions to sustain increases in physical activity in middle-aged adults in deprived urban neighbourhoods. Health Technol Assess 2014;18:1-210.

53 Sevick MA, Napolitano MA, Papandonatos GD, et al. Cost-effectiveness of alternative approaches for motivating activity in sedentary adults: results of Project STRIDE. Prev Med 2007;45:54-61.

54 Bertram MY, Lim SS, Barendregt JJ, et al. Assessing the cost-effectiveness of drug and lifestyle intervention following opportunistic screening for pre-diabetes in primary care. Diabetologia 2010;53:875-81.

55 Neumann A, Schwarz P, Lindholm L. Estimating the cost-effectiveness of lifestyle intervention programmes to prevent diabetes based on an example from Germany: Markov modelling. Cost Eff Resour Alloc 2011;9:17

56 Robroek SJ, Polinder S, Bredt FJ, et al. Cost-effectiveness of a long-term Internet-delivered worksite health promotion programme on physical activity and nutrition: a cluster randomized controlled trial. Health Educ Res 2012;27:399-410.

57 van Keulen HM, Bosmans JE, van Tulder MW, et al. Cost-effectiveness of tailored print communication, telephone motivational interviewing, and a combination of the two: results of an economic evaluation alongside the Vitalum randomized controlled trial. Int J Behav Nutr Phys Act 2010;7:64

58 Gusi N, Reyes MC, Gonzalez-Guerrero JL, et al. Cost-utility of a walking programme for moderately depressed, obese, or overweight elderly women in primary care: a randomised controlled trial. BMC Public Health 2008;8:231.

59 Stevens W, Hillsdon M, Thorogood M, et al. Cost-effectiveness of a primary care based physical activity intervention in 45-74-year-old men and women: a randomised controlled trial. Br J Sports Med 1998;32:236-41.

60 Hogg WE, Zhao $X$, Angus $D$, et al. The cost of integrating a physical activity counselor in the primary health care team. J Am Board Fam Med 2012;25:250-2

61 Speck BJ, Looney SW. Effects of a minimal intervention to increase physical activity in women: daily activity records. Nurs Res 2001;50:374-8.

62 Amarasinghe AK. Cost-effectiveness implications of GP intervention to promote physical activity: evidence from Perth, Australia. Cost Eff Resour Alloc 2010;8:10.

63 Bravata DM, Smith-Spangler C, Sundaram V, et al. Using pedometers to increase physical activity and improve health: a systematic review. JAMA 2007;298:2296-304.

64 Shemilt I, Mugford M, Byford S, et al. Incorporating economics evidence. In: Higgins JP, Green S, eds. Cochrane handbook for systematic reviews of interventions Version 5.1.0 [updated Mar 2011]. The Cochrane Collaboration, 2011. Chapter 15. http://handbook.cochrane.org/

65 Robberstad B. QALYs vs DALYS vs LYs gained: what are the differences, and what difference do they make for health care priority setting? Nor Epidemiol 2005;15:183-91.

66 Murray CJ, Acharya AK. Understanding DALYs (disability-adjusted life years). J Health Econ 1997;16:703-30.

67 Buxton MJ, Drummond MF, Van Hout BA, et al. Modelling in economic evaluation: an unavoidable fact of life. Health Econ 1997;6:217-27.

68 Wolfenstetter SB, Wenig CM. Economic evaluation and transferability of physical activity programmes in primary prevention: a systematic review. Int J Environ Res Public Health 2010;7:1622-48.

69 Hoffmann TC, Glasziou PP, Boutron I, et al. Better reporting of interventions: template for intervention description and replication (TIDieR) checklist and guide. BMJ 2014;348:g1687.

70 Postma MJ, de Vries R, Welte R, et al. Health economic methodology illustrated with recent work on Chlamydia screening: the concept of extended dominance. Sex Transm Infect 2008;84:152-4.

71 National Institute for Health and Clinical Excellence (NICE). Guide to the methods of technology appraisal. London: NICE, June 2008. 